\title{
Comportamiento térmico de mampostería de bloques de yeso hidrófugo con variación del espesor de revestimiento
}

Thermal behaviour of hydrofugated plaster block masonry with variation of coating thickness

Fecha de entrega: 17 de abril 2020

Fecha de aceptación: 25 de agosto 2020

\section{José Reginaldo de Arruda Cavalcanti ${ }^{1}$, Pedro Igor Bezerra Batista ${ }^{1}$, Yêda Vieira Póvoas ${ }^{1}$ y Joaquin Humberto Aquino Rocha ${ }^{2}$}

${ }^{1}$ Escola Politécnica de Pernambuco, Universidade de Pernambuco, 50720-001, Recife, Brasil, josereginaldo20.2014@gmail.com, pedroigorbb@gmail.com (Orcid: 0000-0003-3912-111X), yeda.povoas@gmail.com (Orcid: 0000-0003-1907-415X)

${ }^{2}$ Facultad de Tecnología, Universidad Privada del Valle, Tiquipaya, Bolivia, joaquinaquinorocha@gmail.com

Esta investigación tiene como objetivo analizar la influencia del revestimiento cerámico, simulando el lado externo, y yeso con diferentes espesores, simulando el lado interior de un prototipo de mampostería de bloque de yeso hidrófugo macizo, buscando mejorar el desempeño térmico principalmente en las juntas de asentamiento del bloque. Para ello, se utilizaron dos métodos de ensayos específicos: la cámara térmica y la termografía infrarroja. Se analizó las diferencias de temperatura entre la cara interna y externa por medio de termopares conectados en medio del prototipo y el mapeo del perfil de temperatura en la superficie del revestimiento. Se encontró que la adición de revestimiento de yeso más el cerámico mejora el desempeño térmico del sistema de mampostería, observando que la variación del espesor del mortero de yeso proporciona una ganancia en la resistencia térmica, una reducción en la transmitancia térmica y un aumento en la capacidad térmica.

Palabras clave: desempeño térmico, yeso hidrófugo, cámara térmica, termografía infrarroja, NBR 15220

\section{Introducción}

En regiones de latitudes bajas, la presencia de altas temperaturas y humedad proporciona un aumento en el consumo de energía de los edificios. Según los datos de EPE (2016), entre 1995 y 2014, el consumo de electricidad en Brasil aumentó en un 95\%. Según ABRAVA (2016), los sistemas de construcción contribuyen al consumo de aproximadamente el $50 \%$ de la electricidad utilizada en Brasil, donde una parte significativa de este porcentaje proviene de sistemas dedicados a obtener confort térmico.
The present research has the objective of analysing the influence of the ceramic coatings simulating the external side, and in gypsum, with different thicknesses, simulating the internal side of a masonry prototype of block seal of solid waterproof gypsum, seeking to improve the thermal performance mainly in the block joints of the block. For this, two specific test methods were used: the thermal chamber and the infrared thermography. We sought to analyse the temperature differences between the internal and external surfaces, by means of thermocouples connected in the middle of the prototype and the mapping of the temperature profile on the surface of the coating. It was verified that the addition of gypsum coatings plus ceramic improves the thermal performance of the sealing system. Noting that the thickness variation of gypsum mortar provides a gain in thermal resistance, reduction in thermal transmittance and gain in thermal capacity.

Keywords: thermal performance, waterproof plaster, thermal camera, infrared thermography, NBR 15220 
et al. (2016), la reducción de la carga térmica dentro de los edificios, que proviene en mayor proporción del entorno externo, se puede obtener mediante la aplicación de aislamiento térmico, por ejemplo, recubrimientos opacos de baja conductividad térmica.

El objetivo de este trabajo es analizar el comportamiento térmico de un prototipo de mampostería compuesta por un bloque de yeso hidrófugo macizo con dos tipos de revestimientos: mortero de yeso en el lado interno y cerámica para el lado externo. Se busca realizar una comparación con los parámetros obtenidos por cálculo de acuerdo con la normas NBR 15220-2 (2005) y NBR 15575-4 (2013), además del análisis experimental del prototipo, mediante termografía infrarroja, estableciendo una conexión con los valores obtenidos con termopares de contacto.

\section{El uso del yeso como aislante térmico}

El yeso tiene varias ventajas como un material usado para aislamiento térmico, entre ellas un costo relativamente bajo y un tiempo de ejecución más corto en comparación con otros materiales utilizados como revestimiento, como el cemento Portland. En la construcción civil es ampliamente utilizado en forma de bloques en la ejecución de muros y divisiones internas (Peres et al., 2001). En el año 2015, la producción brasileña de yeso crudo para comercialización y procesamiento alcanzó $3.13 \mathrm{Mt}$, una reducción del 8.5\% con respecto al año anterior, donde el estado de Pernambuco es el principal productor de Brasil, representando el $82.5 \%$ del total producido (ANM, 2018). Según Neves (2011), hay un uso cada vez mayor de este material en edificios de varias plantas en las grandes ciudades del noreste de Brasil e incluso en el interior, precisamente en los estados de Pernambuco, Ceará y Sergipe. Sin embargo, el consumo de yeso estimado es de $7 \mathrm{~kg} /$ habitante/año en Brasil, un valor bajo cuando se compara con Argentina (20 kg/hab/ año), Chile (40 kg/hab/año), Japón ( $80 \mathrm{~kg} / \mathrm{hab} / \mathrm{año})$, EEUU (90 kg/hab/año) y Europa (80 kg/hab/año) (FINEP, 2010).

El yeso es ampliamente utilizado en la producción de bloques prefabricados, donde tienen diferentes características según el aditivo utilizado, siendo reconocidos por el color del bloque: blanco - normal, rosa - resistente al fuego (contiene fibra de vidrio), verde - resistente al agua (adición de un polímero de silicona). También los bloques prefabricados pueden ser utilizados para aislamiento acústico (estructura perforada) y aislamiento térmico (adición de una placa de poliestireno expandido) (Santos, 2008).

Dependiendo de la ubicación y sus características, los bloques de yeso prefabricados permeables se usan exclusivamente como paredes internas $\mathrm{y}$, aquellos con características repelentes al agua, se usan en mampostería externa o en áreas con agua (Costa e Inojosa, 2007). Estos últimos se conocen como bloques hidrófugos y proporcionan un mayor aislamiento térmico (Sobrinho et al., 2010), con un coeficiente de conductividad térmica equivalente a $0.46 \mathrm{~W} /{ }^{\circ} \mathrm{C}$, que indica un material con propiedades de aislante térmico (Incropera y de Witt, 2003).

Los bloques de yeso hidrófugos son similares a los bloques de yeso estándar, excepto por la adición en su composición química de un aditivo repelente al agua. Los bloques repelentes al agua están pigmentados por el color azulado y se usan en la construcción de tabiques internos y externos de áreas húmedas y en la primera fila de áreas secas (PBQP-H, 2017; ITEP, 2007). En Brasil, los bloques hidrófugos tienen una variación de espesor de 50, 70 y 100 $\mathrm{mm}$, con una longitud de $666 \mathrm{~mm}$ y una altura de $500 \mathrm{~mm}$, presentando una capacidad de absorción de agua $\leq 5.0 \%$, definida por el método de prueba descrito en el ítem 11.5 de NBR 16495 (2016). En la Tabla 1 se caracterizan los bloques hidrófugos según criterios normativos.

Tabla 1: Características del bloque de yeso hidrófugo (NBR 16494, 2017)

\begin{tabular}{|l|c|c|}
\hline Características & $\begin{array}{c}\text { Bloque } \\
\text { hidrófugo } 70\end{array}$ & $\begin{array}{c}\text { Bloque } \\
\text { hidrófugo 100 }\end{array}$ \\
\hline Espesor, $\mathrm{mm}$ & 70 & 100 \\
\hline Tipo & Macizo & Macizo \\
\hline Dimensiones, $\mathrm{mm}$ & $666 \times 500$ & $666 \times 500$ \\
\hline Peso medio, $\mathrm{kg}$ & 24 & 34 \\
\hline Peso medio, $\mathrm{kg} / \mathrm{m}^{2}$ & 72 & 102 \\
\hline $\begin{array}{l}\text { Dureza-solidez superficial } \\
\text { (U.S.C.) }\end{array}$ & $\geq 55$ & $\geq 55$ \\
\hline Absorción de agua & $<5 \%$ & $<5 \%$ \\
\hline Resistencia a la flexión, $\mathrm{MPa}$ & $\geq 1.2$ & $\geq 1.5$ \\
\hline
\end{tabular}

Con relación al yeso para revestimiento interno, se constituye principalmente de sulfato de calcio dihidratado, calcinado y reducido a polvo, pudiendo tener adiciones o aditivos en algunos casos (NBR 16494, 2017). Su uso como 
revestimiento en ambientes internos tiene varias ventajas en comparación con otros materiales. Estas ventajas incluyen aislamiento térmico y acústico, estanqueidad, aislamiento contra el fuego, resistencia de desgaste, regularización de superficies y base para acabados decorativos (Carasek, 2008). El yeso tiene un bajo coeficiente de conductividad térmica a comparación de otros materiales, entre 0.25 a $0.50 \mathrm{~W} /\left(\mathrm{m}^{\circ} \mathrm{C}\right)$, esto se debe a la densidad del material, que presenta grandes vacíos en los espacios intercristalinos (Dias y Cincotto, 1998; Peres et al., 2008).

\section{Ensayos de termografía infrarroja y cámara térmica}

La termografía infrarroja es un ensayo que mide la radiación emitida por la superficie de un objeto, que se basa en la perturbación del flujo de calor, generada natural o artificialmente (Kylili et al., 2014). Ello produce desviaciones en la distribución de la temperatura de la superficie del objeto, capturadas por el equipo termográfico, generando una imagen de temperatura, conocida como termograma (Lourenço et al., 2017). El flujo de calor es responsable por las diferencias de temperatura en el termograma, que depende de la condición de exposición del elemento estudiado (Bauer et al., 2015). La termografía infrarroja se utiliza en diversas áreas de ingeniería para monitoreo de equipos y procesos, detección de defectos y estudios de desempeño térmico (Bagavathiappan et al., 2013; Rehman et al., 2016; Lucchi, 2019; O'Grady et al., 2017; Fox et al., 2016).

En el ensayo de la cámara térmica, la temperatura se recolecta a través de termopares ubicados en el objeto. En la cámara hay una fuente de calor que proporciona la energía térmica que es detectada por los termopares (Kylili et al., 2014). Todas las paredes de una cámara térmica en el ambiente calentado deben estar revestidas con material aislante del espesor necesario para evitar la pérdida de calor en el ambiente externo de la cámara durante el ensayo, ya que la temperatura externa siempre es menor que el ambiente calentado dentro de la cámara. Para tener control del ambiente externo es necesario el control climático del ambiente, buscando mantener una temperatura fuera de la cámara térmica cercana de $26^{\circ} \mathrm{C}$. Varios resultados se han obtenido en investigaciones recientes utilizando cámaras térmicas en mampostería de yeso acartonado (da Silva et al., 2013; Ferreira et al., 2016).

\section{Metodología}

Para lograr el objetivo de este estudio, primeramente, fue confeccionado un prototipo de mampostería con bloque de yeso hidrófugo macizo denominado $\mathrm{GH} 100+$, donde: $\mathrm{GH}$ es el acrónimo para yeso impermeable, 100 es el grosor en milímetros y + es la indicación de sólidos. Posteriormente, se revistió con cerámica para simular el lado externo y con yeso de diferentes espesores, 0.5 y $1.0 \mathrm{~cm}$, para simular el lado interno de una mampostería. Ya Batista (2019) desarrolló experimentalmente un prototipo similar con bloques de yeso hidrófugo.

Se utilizó el bloque de yeso hidrófugo, material que ha estado reemplazando tecnologías tradicionales como bloques de hormigón y cerámica para mamposterías. Esto se debe a las ventajas que ofrece, tales como menor tiempo de ejecución, flexibilidad de diseño, mejor rendimiento termoacústico, comodidad, entre otros (Santana et al., 2019). La norma NBR 16494 (2017) proporciona características (Tabla 1) y lineamientos para el uso de bloques de yeso hidrófugo en mampostería vertical.

Considerando que, el mayor consumo de energía se da en regiones con presencia de altas temperaturas y humedad, Zona Bioclimática 8 según la NBR 15220-3 (2005), se utilizó cerámica como revestimiento externo. Además que, en estas regiones el uso de cerámica como revestimiento externo es bastante común (Costa y Silva, 2001). Respecto al recubrimiento de yeso, la NBR 13867 (1997) no recomienda un espesor específico; no obstante, varios autores recomiendan diferentes espesores en el rango de 0.1 a $1.0 \mathrm{~cm}$, valores que dependen del trabajo a ser realizado (Rocha et al., 2004; Yazigi, 2006; Maeda y Souza, 2003). En este sentido, se optó por analizar dos espesores de yeso, 0.5 y $1.0 \mathrm{~cm}$. Para el estudio de su comportamiento térmico, fue colocado en una cámara térmica para su calentamiento y fue utilizada una cámara termográfica para verificar la distribución de la temperatura a lo largo del prototipo. Finalmente, los parámetros térmicos se calcularon de acuerdo con la NBR 15220-2 (2005).

\section{Confección de prototipos}

Primero, fue necesario preparar el bloque de yeso macizo de $100 \mathrm{~mm}$ de espesor (GH 100+), donde se demarcaron las regiones de corte, como se muestra en la Figura 1a, para la producción de un prototipo de mampostería vertical 
de $42 \times 42 \mathrm{~cm}^{2}$. Se cortaron tres bloques, dos de $21 \times 21$ $\mathrm{cm}^{2}$ y uno de $42 \times 21 \mathrm{~cm}^{2}$. Los cortes se hicieron para dejar los extremos macho - hembra, permitiendo que las piezas se pegaran con yeso cola (Figura 1b). Según Souza (2009), el yeso cola tiene una resistencia a la tracción superior al bloque, proporcionando una excelente adherencia y una perfecta unión de las partes.
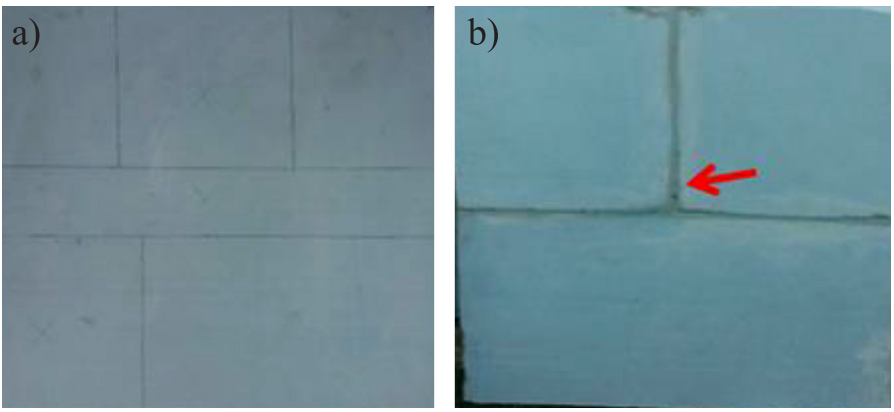

Figura 1: Prototipo GH 100+, a) marcación de los cortes para la confección del prototipo y b) montaje del prototipo de 42 × 42 $\mathrm{cm}^{2}$ destacando la junta con la flecha roja

\section{Ejecución del revestimiento}

La segunda etapa del desarrollo de la investigación consistió en la aplicación de dos revestimientos: placas de cerámica de $10 \times 10 \mathrm{~cm}^{2}$ (simulando el exterior) y yeso de 2 capas (simulando el interior), cada una de $0.5 \mathrm{~cm}$ de espesor. Para el revestimiento cerámico, Weber (2018) recomienda el uso de adhesivos u otros pegamentos que no contengan cemento Portland para el colocado de las placas cerámicas sobre el bloque de yeso. En esta investigación se utilizó yeso cola en la proporción de $1 \mathrm{~kg} / 700 \mathrm{ml}$ de agua, según lo recomendado por el fabricante. Después de 7 días de la colocación, se aplicó la lechada de rejunta y se esperó más de 15 días para evitar la aparición de fisuras durante el calentamiento del sistema (Figura 2a).

El revestimiento de yeso se aplicó en dos capas para lograr
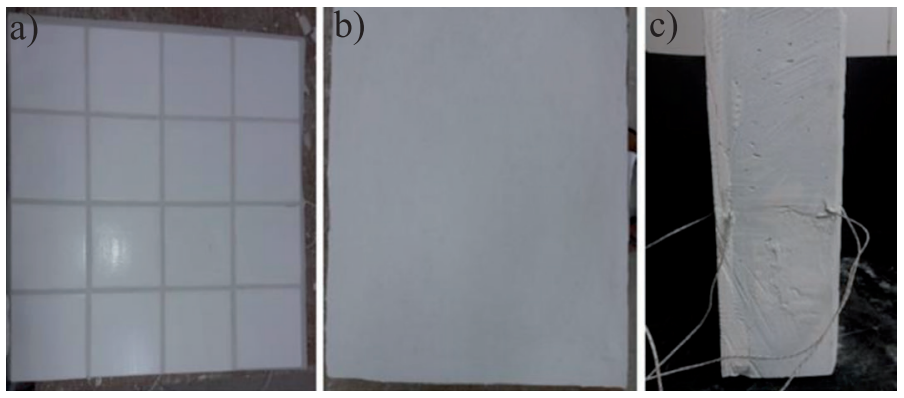

Figura 2: Prototipo de la mampostería: a) revestimiento cerámico, b) revestimiento de yeso y c) corte vertical del prototipo espesores de 0.5 y $1.0 \mathrm{~cm}$ (Figura 2b). Después de la ejecución de la primera capa, se esperó un período de 7 días para verificar el comportamiento térmico del prototipo. Luego se aplicó la segunda capa y se esperaron otros 7 días para la segunda verificación. La Figura 2c muestra el posicionamiento de los dos revestimientos, donde a la derecha está la placa cerámica y a la izquierda el yeso. En el centro está la mampostería GH 100+.

\section{Ensayo de cámara térmica}

Para el ensayo de verificación del comportamiento térmico se usó una cámara térmica en las dimensiones de 43 x 40 x $43 \mathrm{~cm}^{3}$ (Figura 3). La fuente de calor era una lámpara de $250 \mathrm{~W}$ ubicada en la región central del interior, a $11.5 \mathrm{~cm}$ del elemento de prueba. Para fines de recolección de datos (temperatura), se aplicaron dos termopares al interior (lado caliente) y externo (lado frío) de la cámara. La medida de control impuesta fue la regulación térmica del ambiente externo, posibilitando simular un clima más frío, alrededor de $26^{\circ} \mathrm{C}$.

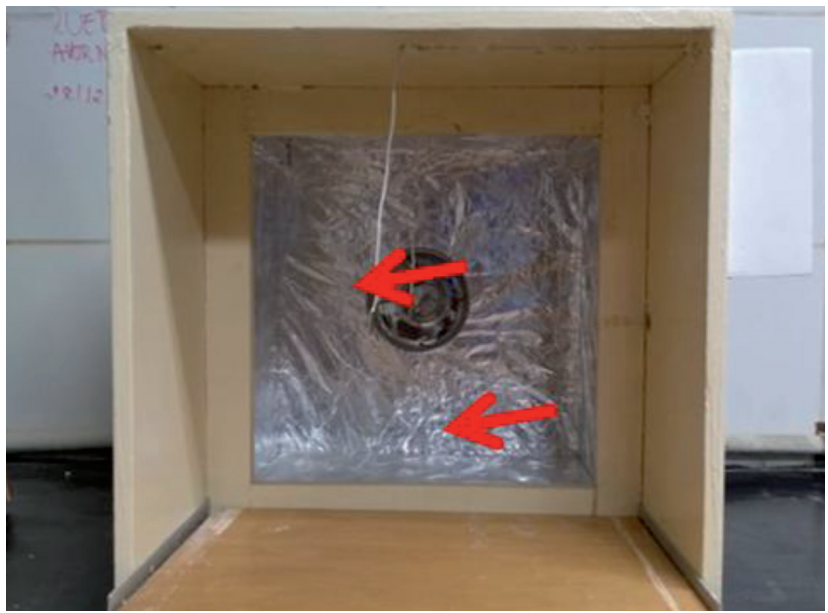

Figura 3: Cámara térmica con lámpara destacando con flechas la fuente de calor y termopar

Según Silva et al. (2012), para obtener datos más representativos, la ubicación del termopar debería estar en el centro del objeto estudiado, donde recibe una mayor concentración de calor de la fuente térmica. Para esta investigación, se optó por colocar los termopares en el centro del prototipo, uno en la cara expuesta al calor (lado caliente) y otro en la cara opuesta (lado frío), fijados por cintas metálicas. Para medir la temperatura de calentamiento, los termopares se conectaron a un termómetro digital de cuatro canales MT-1044 - MINIPA® 
programado para registrar valores en intervalos de un minuto. El ensayo tuvo una duración de 6 horas.

La Figura 4a indica la dirección del flujo de calor que actúa sobre el lado caliente, compuesto por el revestimiento cerámico, y en el lado frío, compuesto por el revestimiento de yeso, siguiendo un modelo similar al de da Silva et al. (2013). Se utilizó el prototipo de mampostería y la verificación del desempeño del sistema de mampostería similar al de Ferreira et al. (2016). La Figura 4b muestra el prototipo después de la aplicación de los revestimientos y listo para el ensayo. Es posible identificar la ubicación de los termopares en el centro del prototipo.

a)

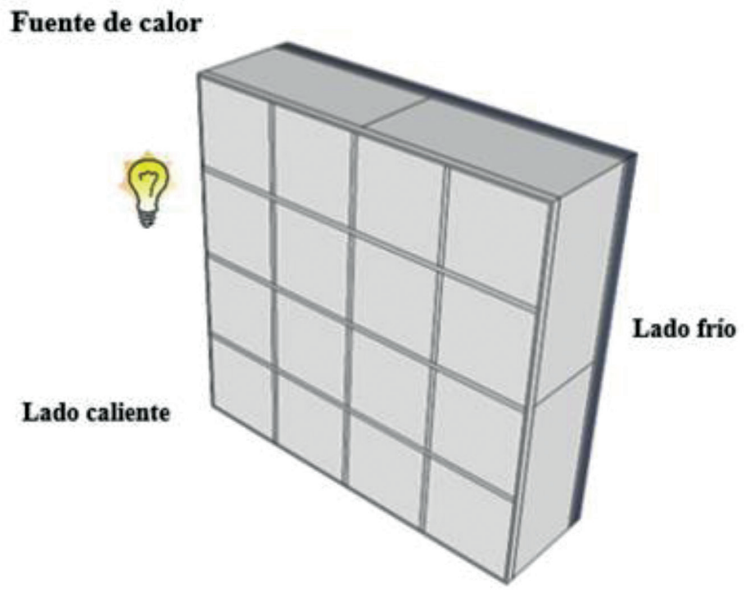

b)

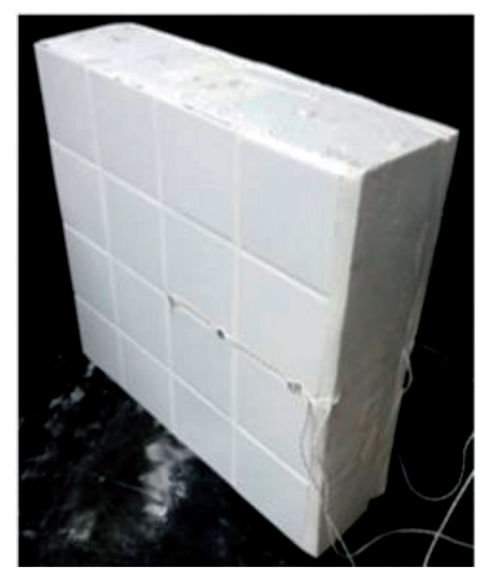

Figura 4: Modelo esquemático del prototipo: a) indicación de la fuente de calor y b) localización de los termopares

Para facilitar la comprensión del análisis, la nomenclatura se adoptó de acuerdo con el orden del ensayo:

Caso 1: prototipo sin revestimiento (Figura 5a);

Caso 2: prototipo con revestimiento cerámico en el lado caliente y $0.5 \mathrm{~cm}$ de revestimiento de yeso en el lado frío (Figura 5b); y
Caso 3: prototipo con revestimiento cerámico en el lado caliente y $1.0 \mathrm{~cm}$ de revestimiento de yeso en el lado frío (Figura 5c).

En cuanto a los termopares, $\mathrm{CH} 1$ y $\mathrm{CH} 3$ se ubicaron en el lado frío, mientras que los termopares $\mathrm{CH} 2$ y $\mathrm{CH} 4$ se ubicaron en el lado caliente (Figura 5). La nomenclatura general se detalla en la Tabla 2.

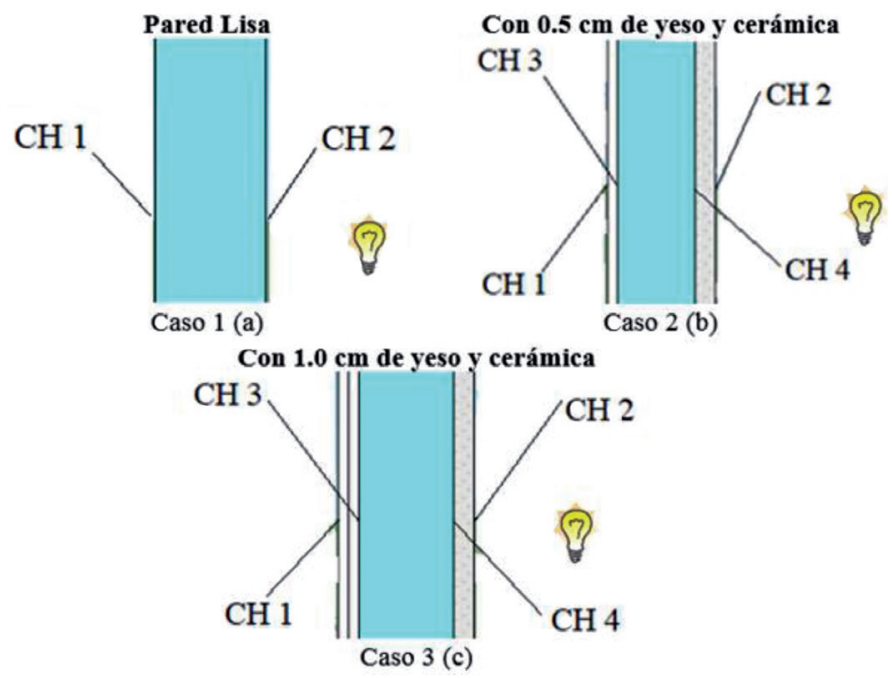

Figura 5: Orden de los ensayos, posición de los termopares e indicación de la localización de la fuente de calor para: a) Caso 1, b) Caso 2 y c) Caso 3

Tabla 2: Resumen de la posición de los termopares

\begin{tabular}{|c|c|l|}
\hline Caso & $\begin{array}{c}\text { Termopar } \\
\text { utilizado }\end{array}$ & \multicolumn{1}{c|}{ Significado } \\
\hline \multirow{2}{*}{1} & CH 1 & Lado frío \\
\cline { 2 - 3 } & CH 2 & Lado caliente \\
\hline \multirow{4}{*}{2} & CH 1 & Lado frío (sobre el revestimiento de yeso) \\
\cline { 2 - 3 } & CH 2 & $\begin{array}{l}\text { Lado caliente (sobre el revestimiento } \\
\text { cerámico) }\end{array}$ \\
\cline { 2 - 3 } & CH 3 & $\begin{array}{l}\text { Lado frío (entre el revestimiento de yeso y el } \\
\text { bloque GH 100+) }\end{array}$ \\
\cline { 2 - 3 } & CH 4 & $\begin{array}{l}\text { Lado caliente (entre el revestimiento cerámico } \\
\text { y el bloque GH 100+) }\end{array}$ \\
\hline \multirow{4}{*}{3} & CH 1 & Lado frío (sobre el revestimiento de yeso) \\
\cline { 2 - 3 } & CH 2 & $\begin{array}{l}\text { Lado caliente (sobre el revestimiento } \\
\text { cerámico) }\end{array}$ \\
\cline { 2 - 3 } & CH 3 & $\begin{array}{l}\text { Lado frío (entre el revestimiento de yeso y el } \\
\text { bloque GH 100+) }\end{array}$ \\
\cline { 2 - 3 } & CH 4 & $\begin{array}{l}\text { Lado caliente (entre el revestimiento cerámico } \\
\text { y el bloque GH 100+) }\end{array}$ \\
\hline
\end{tabular}

\section{Temperatura a lo largo del prototipo mediante termografía intrarroja}

El calentamiento del prototipo fue acompañado con una cámara termográfica en intervalos de una hora. Durante el calentamiento, los termogramas se tomaron solo desde 
el exterior de la cámara térmica (lado frío). El proceso de enfriamiento se registró después de retirar el prototipo de la cámara térmica, donde se generaron termogramas en intervalos de 30 min. Para ello, se utilizó una cámara termográfica FLIR, modelo E60. Los termogramas obtenidos se procesaron asignando un color a cada temperatura (Usamentiaga et al., 2014). La selección cuidadosa del valor de emisividad fue necesaria para simplificar la interpretación de la imagen térmica (Barreira et al., 2017). Según Incropera y de Witt (2003), el valor de la emisividad varía según el tipo de material, y existen varios métodos de prueba posibles, como el método de cinta negra (Lourenço et al., 2017). Para esta investigación, se obtuvieron valores de emisividad para el yeso y la placa cerámica de 0.95 y 0.87 , respectivamente. A través de estos valores, las imágenes térmicas se ajustaron utilizando el software FLIR TOOLS ${ }^{\circledR}$, donde se utilizó el rango de $23^{\circ} \mathrm{C}$ (temperatura más baja) a $39^{\circ} \mathrm{C}$ (temperatura más alta) para mejorar la diferencia entre los termogramas tomados.

\section{Cálculo de parámetros térmicos}

Para calcular los parámetros térmicos del sistema de mampostería se utilizó el método presente en NBR 15220-2 (2005), donde se obtuvo la mayoría de los datos de entrada: conductividad térmica, calor específico y densidad de masa aparente de los materiales utilizados. La densidad de masa aparente de los materiales se determinó en laboratorio utilizando muestras tomadas del stock disponible. La Tabla 3 muestra los valores obtenidos.

Tabla 3: Datos de los materiales utilizados

\begin{tabular}{|l|c|c|c|}
\hline Material & $\begin{array}{c}\text { Conductividad } \\
\text { térmica, } \\
\mathrm{W} /(\mathrm{mK})\end{array}$ & $\begin{array}{c}\text { Calor } \\
\text { específico, } \\
\mathrm{kJ} /(\mathrm{kgK})\end{array}$ & $\begin{array}{c}\text { Densidad de } \\
\text { masa aparente, } \\
\mathrm{kg} / \mathrm{m}^{3}\end{array}$ \\
\hline $\begin{array}{l}\text { Placa de GH } \\
100+\end{array}$ & 0.35 & 0.84 & 1084 \\
\hline Cerámica & 1.05 & 0.92 & 3000 \\
\hline Junta & 1.15 & 1.00 & 1980 \\
\hline $\begin{array}{l}\text { Revestimiento } \\
\text { en yeso }\end{array}$ & 0.70 & 0.84 & 1100 \\
\hline
\end{tabular}

\section{Análisis de resultados}

\section{Temperatura del prototipo durante el ensayo en la cámara térmica}

De los resultados presentados en la Tabla 4 se evidenció que, en el lado frío del prototipo, hasta el período de una hora, no presentó cambios significativos en relación con la temperatura. El Caso $3 \mathrm{CH} 3$ presentó una variación de $1.4^{\circ} \mathrm{C}$ en este intervalo. Sin embargo, después de 2 hrs, hubo un crecimiento de la misma. El Caso 1 mostró la mayor variación de temperatura, alrededor de $9.5^{\circ} \mathrm{C}$ en el periodo de $6 \mathrm{hrs}$ del ensayo, mientras que el Caso 2 y el Caso 3 presentaron una reducción de $7^{\circ} \mathrm{C}(22 \%)$ y $6.5^{\circ} \mathrm{C}(28 \%)$ en comparación con el Caso 1 . Al comparar el termopar $\mathrm{CH} 3$ en los Casos 2 y 3, se observó una reducción de $1.0^{\circ} \mathrm{C}$ y $0.5^{\circ} \mathrm{C}$, respectivamente, en relación con el termopar $\mathrm{CH} 1$ del Caso 1. Los Casos 2 y 3 en relación con el termopar $\mathrm{CH} 3$ mostraron una diferencia de $0.5^{\circ} \mathrm{C}$, probablemente debido a las condiciones del clima controlado del ambiente, provocando esa diferencia en la variación de la temperatura del lado frío durante el ensayo de calentamiento.

Tabla 4: Variación de la temperatura a cada hora en relación al calentamiento del lado frio del prototipo

\begin{tabular}{|c|c|c|c|c|c|}
\hline \multirow{2}{*}{$\begin{array}{c}\text { Hora } \\
\text { hh:mm }\end{array}$} & \multicolumn{5}{|c|}{ Temperatura, ${ }^{\circ} \mathrm{C}$} \\
\cline { 2 - 6 } & $\begin{array}{c}\text { Caso 1 } \\
\text { CH 1 }\end{array}$ & $\begin{array}{c}\text { Caso 2 } \\
\text { CH 1 }\end{array}$ & $\begin{array}{c}\text { Caso 2 } \\
\text { CH 3 }\end{array}$ & $\begin{array}{c}\text { Caso 3 } \\
\text { CH 1 }\end{array}$ & $\begin{array}{c}\text { Caso 3 } \\
\text { CH 3 }\end{array}$ \\
\hline $00: 00$ & 26.3 & 26.8 & 27.3 & 25.8 & 25.7 \\
\hline $01: 00$ & 27.0 & 26.9 & 27.4 & 26.7 & 27.1 \\
\hline $02: 00$ & 29.9 & 28.5 & 29.3 & 27.8 & 28.6 \\
\hline $03: 00$ & 31.8 & 30.3 & 31.4 & 29.4 & 30.6 \\
\hline $04: 00$ & 33.4 & 31.6 & 33.0 & 30.7 & 32.3 \\
\hline $05: 00$ & 34.6 & 32.7 & 34.3 & 31.7 & 33.7 \\
\hline $06: 00$ & 35.8 & 33.8 & 35.8 & 32.3 & 34.7 \\
\hline Variación & 9.5 & 7.0 & 8.5 & 6.5 & 9.0 \\
\hline
\end{tabular}

La Figura 6 muestra la evolución del calentamiento del lado frío, donde es posible observar la variación en el comportamiento del prototipo de acuerdo con la presencia de los revestimientos. En la primera hora la temperatura no varió mucho, pero después de este período, hay un crecimiento de la misma. Se observó que después de completar $6 \mathrm{hrs}$ del ensayo, la temperatura promedio en el lado frío alcanzó el valor máximo. El Caso 1 mostró el mayor crecimiento de temperatura en la región cercana a la junta de unión del bloque, considerando que esta región es un punto crítico para el confort térmico (Poças, 2008). En los Casos 2 CH 1 y $3 \mathrm{CH} \mathrm{1,} \mathrm{una} \mathrm{mejora} \mathrm{puede}$ ser percibida, según el comportamiento que se muestra en la Figura 6, indicando una reducción de aproximadamente $2^{\circ} \mathrm{C}$ después de $6 \mathrm{hrs}$ en comparación con el Caso 1. El 
Caso $2 \mathrm{CH} 3$ y $3 \mathrm{CH} 3$ presentaron un comportamiento similar en relación con el Caso $1 \mathrm{CH} 1$.

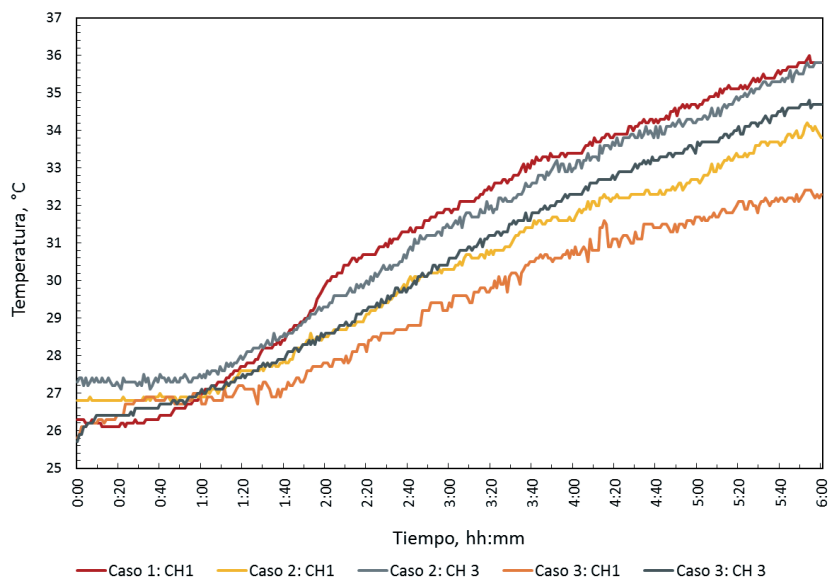

Figura 6: Variación de la temperatura en el calentamiento del lado frío

La variación de temperatura en el lado frío se puede explicar por la transferencia de calor existente en el sistema. En el lado externo (cerámica), existe calentamiento por convección con el aire y radiación por la lámpara de 250 W. Parte de la radiación es reflectada y otra absorbida; la transmisión es cero, una vez que el prototipo analizado es un cuerpo opaco. La radiación absorbida se transmite por el prototipo por conducción calentándolo, Figura 6 . El calentamiento toma un periodo aproximado de una hora para influenciar en la temperatura del lado frío. En el Caso 1, la transferencia de calor es directa. Sin embargo, la presencia de cerámica (Casos 2 y 3) interrumpe la transferencia de calor directa, puesto que la cerámica tiene diferente conductividad térmica (Tabla 3) y, para el mismo tiempo y carga térmica, las temperaturas son menores ( $\mathrm{CH} 3)$ que el Caso $1(\mathrm{CH} 1)$, Figura 6 y Tabla 4. Y para el revestimiento de yeso, la transferencia de calor por conducción se ve limitada por cada capa de yeso adicionada, por eso que la temperatura superficial del Caso $3(\mathrm{CH} 1)$ es menor que el Caso 2 ( $\mathrm{CH} 2)$.

La Tabla 5 muestra el comportamiento del enfriamiento del prototipo en el lado frío. Cabe destacar que, después de apagar la fuente de calor, tardó unos 30 min en reducir la temperatura. Alrededor de $2 \mathrm{hrs}$, según el termopar $\mathrm{CH}$ 1 , el Caso 1 sufrió una pérdida de calor de $3^{\circ} \mathrm{C}$, y para el Caso 2, hubo una reducción de $1.2^{\circ} \mathrm{C}$. En el Caso 3 hubo una reducción de $1.1^{\circ} \mathrm{C}$. Estos resultados indican que hubo una reducción del $60 \%$ en la pérdida de calor para el Caso 2 y del 63\% para el Caso 3, lo que representa una considerable ganancia de confort. Se observa además que para el termopar $\mathrm{CH} 3$ la reducción fue de $1.6^{\circ} \mathrm{C}$ en ambos casos.

Tabla 5: Variación de temperatura a cada media hora en relación al enfriamiento del lado frío del prototipo

\begin{tabular}{|c|c|c|c|c|c|}
\hline \multirow{2}{*}{$\begin{array}{c}\text { Hora } \\
\text { hh:mm }\end{array}$} & \multicolumn{5}{|c|}{ Temperatura, } \\
\cline { 2 - 6 } & $\begin{array}{c}\text { Caso 1 } \\
\text { CH 1 }\end{array}$ & $\begin{array}{c}\text { Caso 2 } \\
\text { CH 1 }\end{array}$ & $\begin{array}{c}\text { Caso 2 } \\
\text { CH 3 }\end{array}$ & $\begin{array}{c}\text { Caso 3 } \\
\text { CH 1 }\end{array}$ & $\begin{array}{c}\text { Caso 3 } \\
\text { CH 3 }\end{array}$ \\
\hline $00: 00$ & 35.9 & 33.9 & 35.7 & 32.3 & 34.8 \\
\hline $00: 30$ & 36.7 & 34.4 & 36.1 & 32.3 & 35.0 \\
\hline $01: 00$ & 35.9 & 34.4 & 36.2 & 32.7 & 34.9 \\
\hline $01: 30$ & 34.7 & 33.6 & 35.0 & 32.0 & 34.0 \\
\hline $02: 00$ & 32.9 & 32.7 & 34.1 & 31.2 & 33.2 \\
\hline Variación & -3.0 & -1.2 & -1.6 & -1.1 & -1.6 \\
\hline
\end{tabular}

Una vez apagada la fuente de calor, el proceso de transferencia de calor continúa durante aproximadamente 30 min (Figura 7). Se observa que el comportamiento presentado por enfriamiento para los casos analizados fue similar, observando que el Caso 1 perdió calor más rápido, estabilizándose alrededor de $32^{\circ} \mathrm{C}$, mientras que para los Casos 2 y 3, al analizar el termopar CH 1, se observa que la pérdida de calor es pequeña, especialmente para el Caso 3, que probablemente se debe a la presencia de una cubierta de yeso de $1 \mathrm{~cm}$ de espesor.

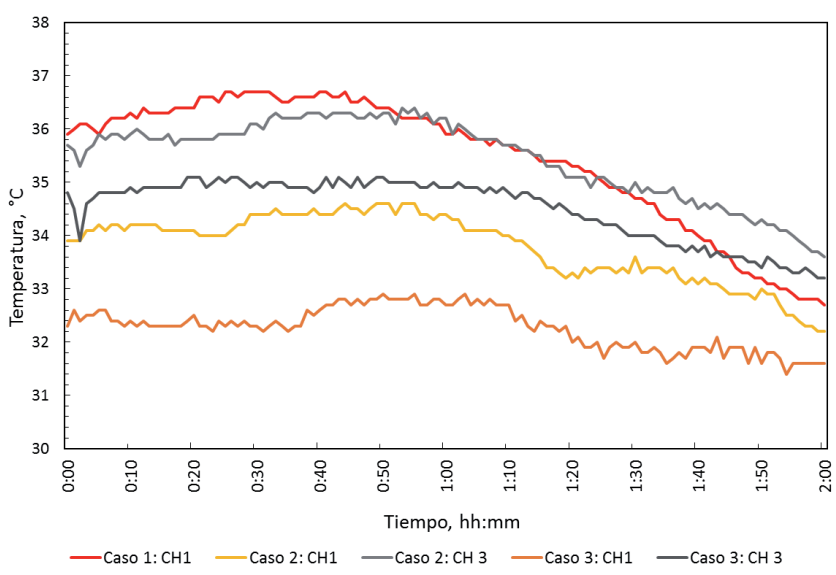

Figura 7: Enfriamiento del lado frío del prototipo en relación al tiempo de análisis

Este comportamiento se debe al equilibrio térmico con el ambiente por el mecanismo de convección. La superficie externa calentada por la lámpara de $250 \mathrm{~W}$ emite radiación calentando el ambiente de la caja térmica, lo que evita que la temperatura baje rápidamente. El calor se mantiene por un periodo de $30 \mathrm{~min}$. En el lado frío, el comportamiento es 
diferente entre el Caso 1 y los Casos 2 y 3 . En el Caso $1 \mathrm{CH}$ 1 la variación es mayor, esto se debe que el prototipo solo consta del bloque $\mathrm{GH} 100+$, por tanto, el equilibrio térmico por convección con el ambiente se realiza de forma más rápida; indicando mayor difusividad térmica (velocidad de variación de temperatura) que los otros sistemas, ya que el bloque GH 100+ tiene mayor conductividad térmica y menor calor específico (Tabla 3). En los Casos $2 \mathrm{CH} 1$ y 3 CH 1 la variación es similar, esto se debe al mismo material del recubrimiento. El equilibrio térmico por convección con el ambiente es parecido en tiempo. Para CH 3 de los Casos 2 y 3 la variación es igual puesto que la transferencia de calor por conducción del lado caliente es la misma, bloque GH 100+ y cerámica.

\section{Temperatura del prototipo mediante termografía infrarroja}

El análisis de termogramas muestra el proceso de calentamiento en el intervalo de $6 \mathrm{hrs}$ de ensayo para el lado frío (Figuras 8, 9 y 10). La escala térmica se ajustó entre 23 y $39^{\circ} \mathrm{C}$ para permitir la comparación entre ensayos. Para el Caso 1, fue posible notar que en la primera hora hubo una variación significativa de tonalidad, intensificada por la transferencia de calor, siendo que cuanto más claro es el color, más alta es la temperatura. La distribución del calor se dio desde la región central hasta los bordes (Figuras 8a y 8 b). Después de 2 hrs (Figura 8c), se observa colores claros en los bordes, más intensos en la parte superior, en la dirección del asentamiento del bloque, lo que indica probablemente la influencia directa en la distribución de calor en este punto. En consecuencia, con el pasar del tiempo, hubo una expansión de esa región acentuándose en una forma redonda, concentrando gran parte de la energía transferida desde la fuente de calor a la parte central (Figuras 8d, 8e y 8f). Al final de las 6 hrs de calentamiento, cabe destacar que la distribución del calor tiende a ocupar las regiones de la junta del asentamiento, moviéndose desde el centro hacia los bordes izquierdo y derecho del medio hacia arriba (Figura 8g), indicando una posible región crítica para el confort térmico de mampostería en yeso hidrófugo macizo.

Los termogramas del Caso 2 tuvieron una intensidad menor del tono, más claro en comparación con el Caso 1. Se observa que el prototipo comenzó a cambiar el tono en la región central a partir de 2 hrs después del inicio

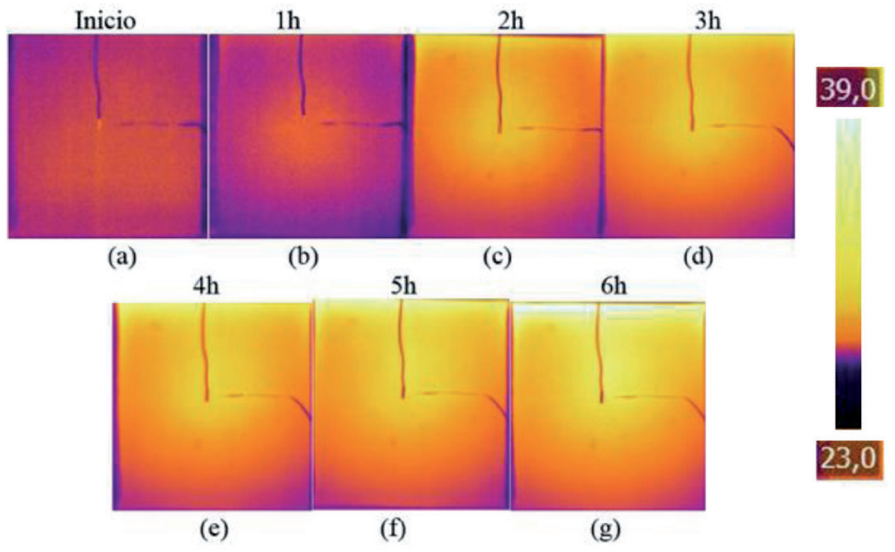

Figura 8: Termogramas del calentamiento del lado frío, Caso 1, para los tiempos en horas: a) 0, b) 1, c) 2, d) 3, e) 4, f) 5 y g) 6

del ensayo, a diferencia del Caso 1 (Figuras 9a, 9b y 9c). Después de este período, se puede observar una ligera variación del tono y la uniformidad de la distribución del tono claro de la superficie, lo que indica una posible mejora en la distribución de calor transferida de la fuente caliente y que el revestimiento de yeso es una posible corrección para el problema presentado en el Caso 1, indicando una mejora en el desempeño térmico del prototipo. Comparando el intervalo final a las $6 \mathrm{hrs}$ de ensayo (Figuras $8 \mathrm{~g}$ y $9 \mathrm{~g}$ ), se observa que ha pasado menos calor.

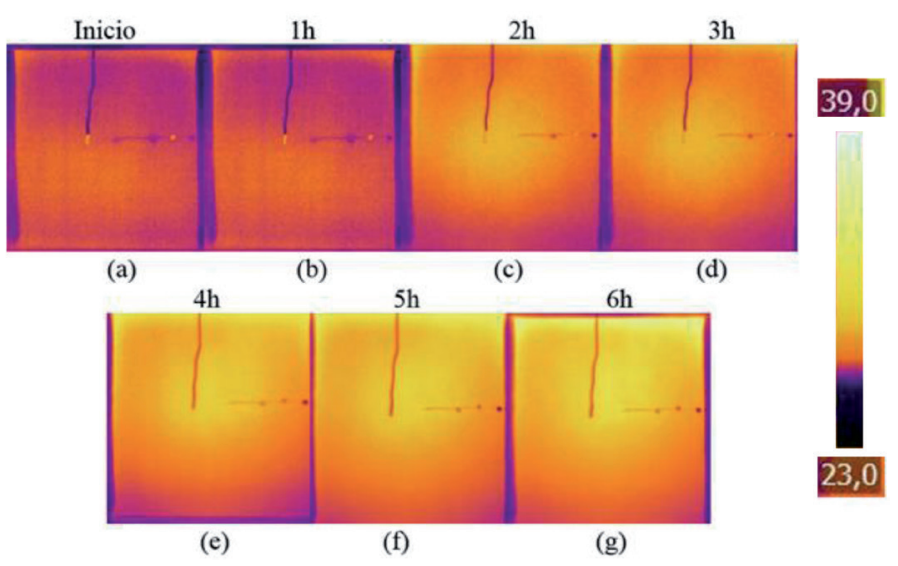

Figura 9: Termogramas del calentamiento del lado frío, Caso 2, para los tiempos en horas: a) 0, b) 1, c) 2, d) 3, e) 4, f) 5 y g) 6

Con respecto al Caso 3, se observó una reducción en el tono en comparación con el Caso 2. El prototipo comenzó a mostrar un cambio de color en la región central 2 hrs después del inicio del ensayo, indicando una mejora con respecto al Caso 1 (Figuras 10a, 10b y 10c). Después de este período (Figura 10d), se observa que la distribución de calor en la superficie es más uniforme y menos intensa que la del Caso 2, lo que indica que el aumento del espesor del 
revestimiento contribuye a mejorar el desempeño térmico de la mampostería al aumentar la resistencia térmica (Tabla 6). Al comparar el intervalo final para los Casos 1, 2 y 3 después de 6 hrs de ensayo (Figuras $8 \mathrm{~g}$, 9g y 10g), se observó que, con la misma carga térmica e intervalo de exposición, el prototipo que presentó mejor desempeño fue el Caso 3.

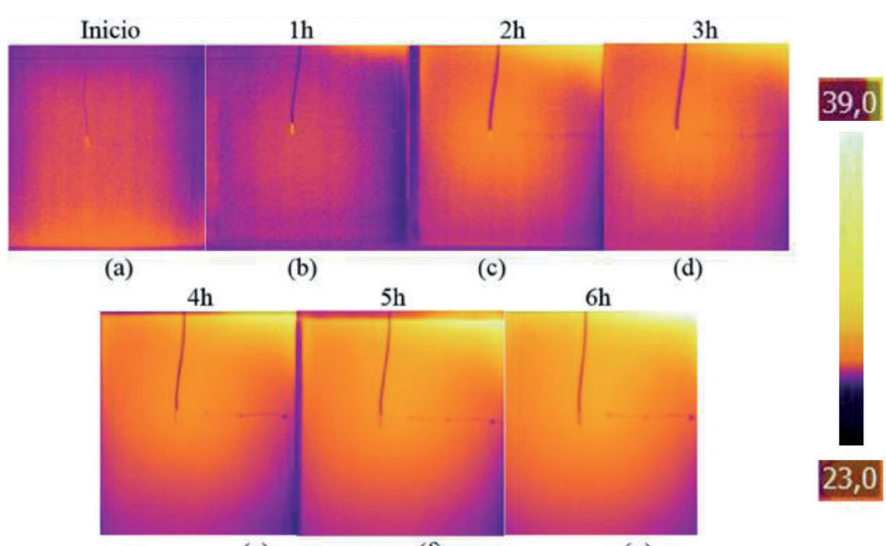

(e)

(f)

(g)

Figura 10: Termogramas del calentamiento del lado frío, Caso 3, para los tiempos en horas: a) 0, b) 1, c) 2, d) 3, e) 4, f) 5, y g) 6

Como se observa en los tres casos presentados, las mayores temperaturas se presentan en la parte central del prototipo, indicando que el calentamiento se da mayormente por la radiación absorbida de la lámpara de $250 \mathrm{~W}$ que por la convección con el aire dentro del prototipo. Aunque también se observa, en horas posteriores, que la parte superior del prototipo presenta mayor temperatura que la parte inferior, esto se debe a la transferencia de calor por convección, ya que los fluidos calientes (aire) se mueven hacia arriba, debido al aumento de volumen (densidad menor). De los termogramas presentados se puede apreciar cualitativamente el calentamiento en el prototipo, verificando la difusividad térmica de cada sistema. Como se explicó en el anterior subtítulo, la mayor difusividad térmica corresponde al Caso 1 y la menor al Caso 3.

\section{Análisisis del cálculo de los parámetros térmicos}

La Tabla 6 muestra los valores obtenidos de los parámetros térmicos, según NBR 15220-2 (2005), para el prototipo con las diferentes configuraciones de revestimiento (Casos $1,2$ y 3$)$.

Según NBR 15220-2 (2005), cuanto mayor sea la resistencia térmica, mejor será el desempeño térmico del sistema. Para los casos con la adición de revestimiento, se puede observar una ganancia de resistencia térmica sobre el prototipo sin la presencia de revestimiento. El Caso 2 presentó el valor de $0.47 \mathrm{~m}^{2} \mathrm{~K} / \mathrm{W}$, que corresponde a un aumento del $2.41 \%$ en comparación con el Caso 1 , y el Caso 3 presentó el valor de $0.48 \mathrm{~m}^{2} \mathrm{~K} / \mathrm{W}$, lo que equivale a una ganancia de $4.17 \%$ sobre el Caso 1 , es decir, el prototipo con mayor espesor de revestimiento resiste mejor el flujo térmico.

Tabla 6: Resistencia térmica total, transmitancia térmica, capacidad térmica y retraso térmico para los casos estudiados

\begin{tabular}{|l|c|c|c|c|}
\hline Casos & $\begin{array}{c}\text { Resistencia } \\
\text { térmica total, } \\
\mathrm{m}^{2} \mathrm{~K} / \mathrm{W}\end{array}$ & $\begin{array}{c}\text { Transmitancia } \\
\text { térmica, } \\
\mathrm{W} /\left(\mathrm{m}^{2} \mathrm{~K}\right)\end{array}$ & $\begin{array}{c}\text { Capacidad } \\
\text { térmica, } \\
\mathrm{kJ} /\left(\mathrm{m}^{2} \mathrm{~K}\right)\end{array}$ & $\begin{array}{c}\text { Retraso } \\
\text { térmico, } \\
\text { horas }\end{array}$ \\
\hline Caso 1 & 0.46 & 2.19 & 91.06 & 3.72 \\
\hline Caso 2 & 0.47 & 2.14 & 108.78 & 3.75 \\
\hline Caso 3 & 0.48 & 2.11 & 113.82 & 3.79 \\
\hline
\end{tabular}

Con respecto a la transmitancia térmica, cuanto menor sea el valor de este parámetro, mejor será el desempeño térmico del componente, ya que el flujo de calor que lo atravesará será menor. El Caso 2 presenta el valor del orden de $2.14 \mathrm{~W} /\left(\mathrm{m}^{2} \mathrm{~K}\right)$, lo que representa una reducción del $2.41 \%$ en comparación con el Caso 1 , y $2.11 \mathrm{~W} /\left(\mathrm{m}^{2} \mathrm{~K}\right)$ para el Caso 3, equivalente a una reducción del 4.17\%, siendo que este último presenta mejor desempeño térmico. Los valores de transmitancia están por debajo de $2.5 \mathrm{~W} /$ $\left(\mathrm{m}^{2} \mathrm{~K}\right)$ presentando desempeño en relación con las paredes externas según el ítem 11.2.1 de NBR 15575-4 (2013).

La capacidad térmica representa la cantidad de calor requerida para variar en $1{ }^{\circ} \mathrm{C}$ en un determinado sistema. Puede notarse que el Caso 2 obtuvo un valor de 108.78 $\mathrm{kJ} /\left(\mathrm{m}^{2} \mathrm{~K}\right)$, lo que representa un aumento del $19.4 \%$ en comparación con el Caso 1, y el caso 3 obtuvo un valor de $113.82 \mathrm{~kJ} /\left(\mathrm{m}^{2} \mathrm{~K}\right)$, que representa una ganancia del $25 \%$ en comparación con el Caso 1. Los valores presentados para la capacidad térmica están por debajo de $130 \mathrm{~kJ} /\left(\mathrm{m}^{2} \mathrm{~K}\right)$, no cumpliendo con el criterio 11.2.2 de NBR 15575-4 (2013).

Según NBR 15220-2 (2015), el retraso térmico representa el tiempo requerido para que la acción térmica de un medio se manifieste en la superficie opuesta, sometida a un régimen periódico de transmisión de calor. Se observó que el prototipo sin recubrimiento (Caso 1) tenía un valor de 3.72 hrs, mayor que la adición de los revestimientos, que fue de 
3.65 hrs para el Caso 2, y de 3.69 hrs para el Caso 3. NBR 15220-2 (2005) señala que, en el caso de un componente formado por diferentes materiales superpuestos en $n$ capas paralelas a las caras (perpendiculares al flujo de calor), el retraso térmico varía según el orden de las capas, lo que puede justificar esta variación en los valores de retraso térmico con la adición de los recubrimientos.

En el presente estudio los resultados están limitados a una prueba. Es necesario la realización de más ensayos considerando otras variables como repetitividad de los datos, espesores intermedios de revestimiento de yeso, diferentes fuentes de calentamiento, entre otros, a fin de tener certeza de la influencia del revestimiento en el comportamiento térmico de mampostería de bloque de yeso; sin embargo, los resultados presentados dan una idea del comportamiento de este que, pueden servir como base de próximas investigaciones.

\section{Conclusiones}

Al analizar los resultados obtenidos con la cámara térmica, se verificó que había una ganancia en el comportamiento térmico del prototipo con la presencia de los revestimientos, indicando la reducción de la carga térmica transferida y la consiguiente ganancia en el confort térmico. Esta ganancia fue mayor cuando se incrementó el espesor del revestimiento de yeso. La influencia se produjo tanto en los procesos de calentamiento como de enfriamiento. Adicionalmente se pudo observar que la variación de temperatura en el calentamiento fue mayor que en el enfriamiento debido a la eliminación de la fuente de calor. El mismo resultado se verificó al analizar los termogramas. Hubo ganancia en el comportamiento térmico en relación con el lado frío por la presencia de los revestimientos de yeso.

Con respecto a los cálculos de los parámetros térmicos, la capacidad térmica del prototipo se incrementó en un $25 \%$ con la aplicación de $1.0 \mathrm{~cm}$ de revestimiento de yeso con cerámica en comparación con el mismo sin recubrimiento.

Por los resultados obtenidos, se puede deducir que las mamposterías de yeso hidrófugo macizo mejoran significativamente el desempeño térmico cuando se aplica un revestimiento de yeso o cerámica. Aun así, es necesaria mayor investigación para generalizar las conclusiones y tener exactitud del comportamiento térmico del yeso hidrófugo.

\section{Referencias}

ABRAVA (2016). O consumo de energia elétrica nas edificações do Brasil. Associação Brasileira de Refrigeração, Ar Condicionado, Ventilação e Aquecimento ABRAVA. http:// abrava.com.br

ANM (2018). Sumário mineral 2016. Departamento Nacional da Produção Mineral. Agência Nacional de Mineração. Brasília, Brasil

Azevedo, C., Santos, I.I. e Marinho, G.S. (2016). Análise de propriedades termofísicas de compósito para isolação térmica. Congresso Nacional de Engenharia Mecânica CONEM, Fortaleza, Brasil

Bagavathiappan, S., Lahiri, B.B., Saravanan, T., Philip, J. and Jayakumar, T. (2013). Infrared thermography for condition monitoring - A review. Infrared Physics \& Technology 60, 35-55 Barreira, E., Almeida, R.M. and Moreira, M. (2017). An infrared thermography passive approach to assess the effect of leakage point in buildings. Energy and Buildings 140, 224-235

Batista, P. (2019). Parâmetros de desempenho térmico de blocos de gesso. MSc. thesis, Escola Politécnica de Pernambuco, Recife, Brasil

Bauer, E., Castro, E.K., Pavon, E. and Oliveira, A.H. (2015). Criteria for application and identification of anomalies on the facades of buildings with the use of passive infrared thermography. $1^{\text {st }}$ International Symposium on Building Pathology. Porto, Portugal

Bezerra, L.A. e Marinho, G.S. (2008). Elementos de alvenaria termo-isolante produzidos com poliestireno expandido reciclado. Mens Agitat 3(2), 17-26

Carasek, H. (2007). Argamassas. Materiais de Construção Civil e Princípios de Ciência e Engenharia de Materiais. Isaia, G.C. (ed.). Instituto Brasileiro do Concreto IBRACON. São Paulo, Brasil

Çengel, Y.A. (2007). Heat and mass transfer: A practical approach. 3rd edition. McGraw-Hill, New York, USA

Costa, A.M. e Inojosa, A.C. (2007). Alvenaria em blocos de gesso. Sistema Construtivo Gypway. Sindicato da Indústria do Gesso SINDUSGESSO, Brasil

Costa e Silva, A.J. (2001). Descolamentos dos revestimentos cerâmicos de fachada na cidade do Recife. MSc thesis, Universidade de São Paulo, Brasil 
da Silva, E.P., de Melo, A.B. e Queiroga, A.B. (2013). Desempenho térmico de vedações: estudo comparativo com blocos de EVA, tijolo cerâmico e gesso acartonado. XII ENCACEncontro Nacional de Conforto no Ambiente Construído e VIII ELACAC-Encontro Latinoamericano de Conforto no Ambiente Construído. Brasília, Brasil

Dias, A. e Cincotto, M. (1995). Revestimento à base de gesso de construção. Boletim Técnico 142. EPUSP. São Paulo, Brasil

EPE (2016). Consumo nacional de energía: 1995-2014. Empresa de Pesquisa Energética EPE. Rio de Janeiro, Brasil

Ferreira, P.R., Henriques, V.M., de Melo, A.B. e Gomes, E.G. (2016). Avaliação do comportamento térmico de paredes monolíticas executadas com diferentes materiais em condições variáveis de oscilações de temperaturas superficiais. XVI Encontro Nacional de Tecnologia do Ambiente Construído, São Paulo, Brasil

FINEP (2010). Financiadora de estudos e projetos (www.finep. gov.br)

Fox, M., Goodhew, S. and Wilde, P. (2016). Building defect detection: External versus internal thermography. Building and Environment 105, 317-331

Incropera, F.P. e de Witt, D.P. (2003). Fundamentos de transferência de calor e de massa. $4^{\mathrm{a}}$ edição. Guanabara Koogan, Rio de Janeiro, Brasil

ITEP (2007). Casas térreas em paredes de alvenaria em blocos de gesso. Manual Construtivo. Recomendações Técnicas ITEP. Recife, Brasil

Kylili, A., Fokaides, P.A., Christou, P. and Kalogirou, S.A. (2014). Infrared thermography (IRT) applications for building diagnostics: a review. Applied Energy 134, 531-549

Lourenço, T., Matias, L. and Faria, P. (2017). Anomalies detection in adhesive wall tiling systems by infrared thermography. Construction and Building Materials 148, 419-428

Lucchi, E. (2018). Applications of the infrared thermography in the energy audit of buildings: A review. Renewable and Sustainable Energy Reviews 82, 3077-3090

Maeda, F.M. e Souza, U.E.L. (2003). Previsão da produtividade da mão-de-obra na execução de revestimento interno em gesso. Boletim técnico 332. EPUSP. São Paulo, Brasil
NBR 13867 (1997). Revestimento interno de paredes e tetos com pasta de gesso - Materiais, preparo, aplicação e acabamento. Associação Brasileira de Normas Técnicas ABNT, Rio de Janeiro, Brasil

NBR 15220-2 (2005). Desempenho térmico de edificações. Parte 2: Método de cálculo da transmitância térmica, da capacidade térmica, do atraso térmico e do fator solar de elementos e componentes construtivos. Associação Brasileira de Normas Técnicas ABNT, Rio de Janeiro, Brasil

NBR 15220-3 (2005). Desempenho térmico de edificações. Parte

3: Zoneamento bioclimático brasileiro e diretrizes construtivas para habitações unifamiliares de interesse social. Associação Brasileira de Normas Técnicas ABNT, Rio de Janeiro, Brasil

NBR 15575-4 (2013). Edifícios habitacionais - Requisitos para sistema de vedação vertical interna e externa - SVVIE. Associação Brasileira de Normas Técnicas ABNT. Rio de Janeiro, Brasil

NBR 16494 (2017). Bloco de gesso para vedação vertical Requisitos. Associação Brasileira de Normas Técnicas ABNT, Rio de Janeiro, Brasil

NBR 16495 (2016). Bloco de gesso para vedação vertical Método de ensaio. Associação Brasileira de Normas Técnicas ABNT, Rio de Janeiro, Brasil

Neves, M.L. (2011). Método construtivo de vedação vertical interna com blocos de gesso. MSc thesis, Escola Politécnica de Pernambuco, Recife, Brasil

O'Grady, M., Lechowska, A.A. and Harte, A.M. (2017). Infrared thermography technique as an in-situ method of assessing heat loss through thermal bridging. Energy and Buildings 135, 20-32

PBQP-H (2017). Programa Brasileiro da qualidade e produtividade do habitat. Sistema Nacional de Avaliações Técnicas SINAT. Brasilia, Brasil, http://pbqp-h.mdr.gov.br/ projetos_sinat.php

Peres, L., Benachour, M. e Santos, V.A. (2008). Gesso: produção e utilização na construção civil. Sebrae, Recife, Brasil

Peres, L.M., Benachour, M. e Santos, V.A. (2001). O Gesso: produção e utilização na construção civil. Bagaço, Recife, Brasil

Poças, J.P. (2008). Estudo do comportamento térmico e mecânico em paredes de alvenaria. MSc thesis, Universidade do Porto, Portugal 
Rehman, S.K.U., Ibrahim, Z., Memon, S.A. and Jameel, M. (2016). Nondestructive test methods for concrete bridges: A review. Construction and Building Materials 107, 58-86

Rocha, E., Medeiros, E., Gabriel, F., Uchôa, J., Marlo, J., Santos, L. e Medeiros, P. (2004). Sistemas de revestimentos-Diagnóstico Local - Brasília/DF. Universidade de Brasília, Brasil

Santana, C.V.D., Póvoas, Y.V., Silva, D.G.C.D. and Miranda Neto, F.D.A. (2019). Recycled gypsum block: development and performance. Ambiente Construído 19(2), 45-58

Santos, R. (2008). Estudo térmico e de materiais de um composto a base de gesso e EPS para construção de casas populares. MSc thesis, Universidade Federal de Rio Grande do Norte, Natal, Brasil

Silva, E.P., Cahino, J.E. e Melo, A.B. (2012). Avaliação do desempenho térmico de blocos EVA. Encontro Nacional de Tecnologia do Ambiente Construído, Juiz de Fora, Brasil

Sobrinho, C.W.A.P., Bezerra, N.M., Costa, T.C.T. e Silva, C.B.A.S. (2010). Divisórias internas de edifícios em alvenaria de blocos de gesso - Vantagens técnicas, econômicas e ambientais. Congresso Internacional de Tecnologia Aplicada para a Arquitetura e Engenharia Sustentáveis. Recife, Pernambuco, Brasil
Souza, A.C. (2009). Análise comparativa de custos de alternativas tecnológicas para construção de habitações populares. MSc thesis, Universidade Católica de Pernambuco, Recife, Brasil

Souza, V.A. (2012). Estudo do comportamento de materiais não convencionais utilizados como revestimentos de parede, visando à redução da carga térmica. MSc thesis, Universidade Federal da Paraíba, João Pessoa, Brasil

Usamentiaga, R., Venegas, P., Guerediaga, J., Vega, L., Molleda, J. and Bulnes, F.G. (2014). Infrared thermography for temperature measurement and non-destructive testing. Sensors 14(7), 12305-12348

Weber (2018). Colagem de cerâmica sobre suportes de gesso em interior. (https://www.weber.com.pt/colagem-e-betumacao-deceramica)

Yazigi, W. (2006). A técnica de edificar. $6^{\text {a }}$ ed. Pini. São Paulo, Brasil 